\title{
Modelling of vertical nano-needles as sensing devices for neuronal signal recordings
}

\author{
Federico Leva ${ }^{1}$, Pierpaolo Palestri ${ }^{2}$ and Luca Selmi ${ }^{1}$ \\ ${ }^{1}$ Dipartimento di Ingegneria Enzo Ferrari, University of Modena and Reggio Emilia, Modena, Italy. \\ ${ }^{2}$ Politechnical Department of Engineering and Architecture, University of Udine, Udine, Italy.
}

This paper reports a design-oriented numerical study of vertical Si-nanowires to be used as sensing elements for the detection of the intracellular electrical activity of neurons. An equivalent lumped-element circuit model is derived and validated by comparison with physics-based numerical simulations. Most of the component values can be identified individually by geometrical and physical considerations. The transfer function and the SNR of the sensor in presence of thermal noise are derived, and the impact of the device geometry is shown.

Index Terms-Neuronal recordings, Nanowires, TCAD simulations, Intracellular sensing, Bio-electronics.

\section{INTRODUCTION}

The design of arrays of passive vertical Si-nanowires (Sinano-needles) fabricated onto low-cost CMOS wafers represents an attractive perspective toward the increasing demand for technologies to reveal the brain operation in real time and for the treatments of brain disorders. CMOS-nanowires integration allows to increase the sensitivity compared to conventional planar electrodes, significantly improving the spatial resolution and the quality of the recorded signal [1], [2]. In this structure the signal amplification can be performed directly on the same CMOS chip hosting the sensing device, thus avoiding inherent parasitic effects of the long interconnect lines from the nano-needles to the pads. Owing to their mature technology, which can translate into faster fabrication and consequently into faster closed-loop in-vitro biocompatibility test, such approach has been pursued by several research groups [2], [3], [4], [5]. The shape of the probing nano-devices ranges from a pure cylindrical nanowire (NW) to a mushroomlike or tapered one, to improve the biocompatibility, the neuron adhesion and the coupling to the neuron [1], [6], [7], [8]. In order to take full advantage of integration, it is important to cooptimize the needle, interconnects and readout designs, which in turn generates a need for increasingly accurate physicsbased models.

This paper presents a hierarchy of integrated nanoneedle models (both numerical and analytical) to study the AC, transient and noise transduction responses. Section II depicts a physic-based parametrized numerical model of vertical Sinanoneedle devices with cylindrical symmetry, simulated using Sentaurus TCAD. Section III introduces two lumped-element equivalent circuit models of the structure of different complexity validated by means of comparison with the numerical TCAD model. Section IV provides expressions of the transfer function between the neuron membrane potential and the input port of the read-out. The SNR at the readout port is computed under the assumption that only thermal noise is affecting the device. Finally, conclusions are given in section V.

\section{Physics-BASED PARAMETRIC FEM MODEL}

The numerical model is built in the Sentaurus TCAD by Synopsis [9], exploiting the material type "freesemiconductor" and the drift diffusion formalism as a mean to emulate the behaviour of the electrolytes inside and outside the neuron, while the phospholipidic membrane is emulated at first order by a lossless insulator layer.

The schematic representation of the system under study is given in Fig. 1. The electrolyte is treated as a semiconductor

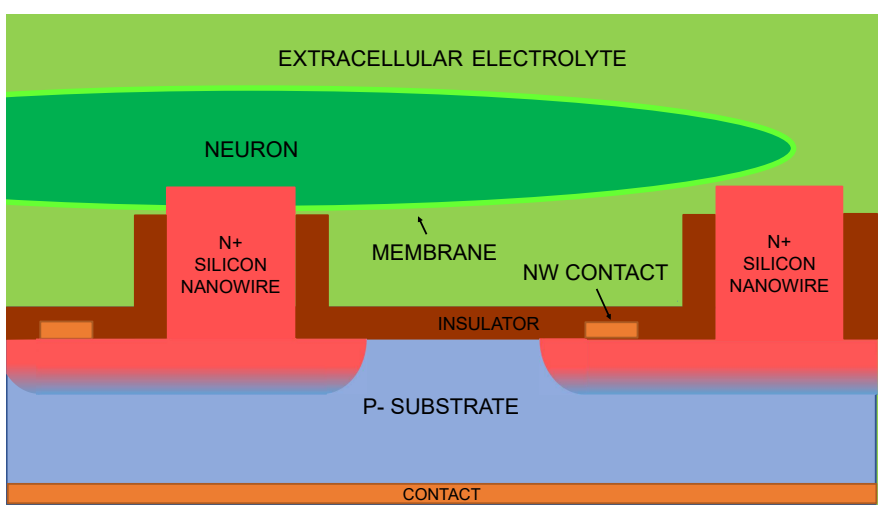

Fig. 1. Schematic representation of the system. The left nanowire is performing an intracellular sensing with nearly ideal insertion contacting with its tip the intracellular electrolyte, while the right one is detached from the needle and lowly coupled to it. The extracellular electrolyte is generically called "electrolyte".

material, where holes and electrons correspond to the dominant cation and anion in the electrolyte. By tailoring the permittivity, the mobility, the bandgap $\left(E_{g}=0 \mathrm{eV}\right)$ and the effective density of states in both the conduction and valence bands equal $\left(N_{C}=N_{V}=N_{a v} c^{0} 10^{-3}\right.$, where $c^{0}$ is the ion molar concentration [10]) one can mimic the electrical behaviour of an electrolyte.

The membrane is modelled as an insulating layer, with a relative permittivity equal to that of the biological neuronal membrane $\left(\epsilon_{r, m e m b} \approx 11\right.$ [11]). We neglect the presence of the ion channels which modulate the membrane conductances. 
Consequently, in our model the well known action potential (AP) neural signal (which alters the intra- and extra- cellular ionic concentrations) is taken as an external boundary condition, and it is applied directly between the external electrolyte contact and the internal neuron contact by means of an independent voltage generator with suitable signal waveform (see dashed-orange line in Fig. 4 below). The underlying idea is to consider only the effects that an AP has on the intra- and extra- cellular electrolytes skipping any detailed physical description of its generation mechanism, since the ultimate goal is to describe how the potential couples to the nanowire and not how it is generated.

Since the electrolytes are emulated by the "freesemiconductor" material, the affinity must be chosen to yield flat energy bands at the equilibrium. The physiological rest membrane potential (approximately $-70 \mathrm{mV}$ ) is superimposed to the AP waveform in the simulations.

Fig.2 shows the physical structure of the studied passive

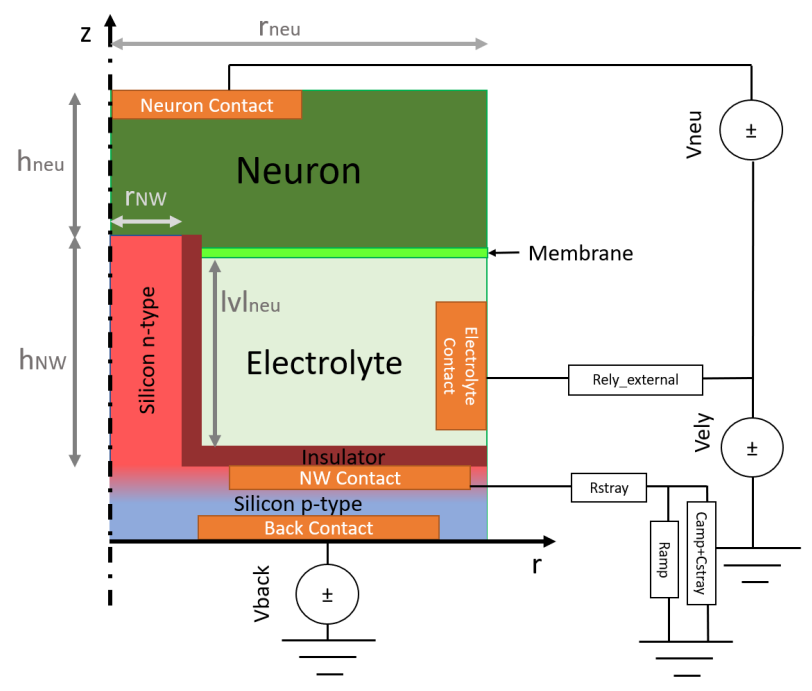

Fig. 2. 2D radial cross section of the nanowire-neuron coupling region used for mixed-mode FEM-circuit simulations in cylindrical coordinates. $V_{\text {neu }}$ is the AP. The contact areas are artificially inflated to make them more visible.

device as 2D cross section that is solved in cylindrical coordinates around the $\mathrm{z}$-axis since the 3D device structure is assumed to have cylindrical symmetry. All the geometrical dimensions are parametrized and their effects on the needle transient and $\mathrm{AC}$ response have been investigated. For the purpose of the following simulations the geometrical design parameter values listed in Table I have been used.

\begin{tabular}{|c|c|c|c|}
\hline nanowire radioius & $r_{N W}$ & $480 \mathrm{~nm}$ & {$[12]$} \\
nanowire height & $h_{N W}$ & $1 \mu \mathrm{m}$ & {$[3]$} \\
neuron radius & $r_{n e u}$ & $5 \mu \mathrm{m}$ & {$[13]$} \\
neuron height & $h_{n e u}$ & $2 \mu \mathrm{m}$ & {$[13]$} \\
neuron z-level & $l v l_{\text {neu }}$ & $40 \mathrm{~nm} ; 1 \mu \mathrm{m}$ & {$[6]$} \\
\hline
\end{tabular}

TABLE I

MAIN GEOMETRICAL DEVICE PARAMETERS ASSUMED IN THIS STUDY

The smooth from red to light-blue grading represents the graded isolation junction between the N-type doped Sinanowire and the the lightly-doped P-type substrate. The vertical position of the membrane can be adjusted to mimic situations where the needle penetrates the neuron to different degrees or even remains at some distance above the needle (no insertion). In order to keep the simulation domain at a manageable size, external components are added to represent the electrolyte between the neuron and the far bulk-electrolyte contact, and the stray resistance and capacitance of the NW contact interconnect. The $R_{a m p} / / C_{a m p}$ parallel represents the input impedance of the readout.

\section{LUMPED-ELEMENT EQUIVALENT CIRCUIT MODEL}

To allow for much faster simulations than the numerical FEM ones, and to easily account for external components (such as the membrane conductances proposed in some membrane models [14], [15]) a lumped element model of the structure has been developed as well, where the nanowire and the electrolyte are represented as distributed $\mathrm{RC}$ networks with parameter values chosen according to the structure physical properties and the geometry. This complete model, shown in Appendix (Fig. A1), has been analyzed in detail for a variety of physical and geometrical characteristics. As an example, Fig. A2 in Appendix shows the degradation of the coupling capacitance for decreasing needle doping and the modest impact of the needle shape. The analysis led to identify a set of optimal parameters for which the model can be simplified with a significant reduction in complexity. This reduced compact model is shown in Fig. 3; the topology is consistent with that of [16].

The relations used to calculate the lumped component values given the geometry and physical properties of the device are shown in Table II. The components which cannot be determined from the geometry, e.g. because of the random nature of the phenomena involved, such as the $R_{\text {njseal }}$ (that accounts for the non-ideal nanowire penetration to the neuron which depends on the neuron-needle adherence) do not appear in the table but are taken from [16].

$\mathrm{AC}$ and transient simulations have been performed to validate the model, and to study the neuron-needle coupling and its dependence on the circuit parameters. For consistency of the model validation phase, all these external components (e.g. the parasitics and the input impedance of the readout) are introduced into the Sentaurus simulations as well, thanks to the mixed-mode device-circuit simulation capability that allows the user to add external lumped elements to the FEM device model.

\section{A. Transient simulations}

Transient simulations have been run where the input signal $\mathrm{V}_{\text {neu }}$ consists of a digitized typical action potential waveform. In both the mixed-mode FEM simulation and the lumpedelement circuit simulation, the potential is applied between the neuron contact $\left(\mathrm{V}_{\text {neu }}\right)$ and the electrolyte contact $\left(\mathrm{V}_{\text {ely }}\right)$ (see Fig. 2 and Fig. 3). Suitable initial conditions on the capacitors eliminate any effect of the slow-charging of the output voltage to the baseline value $(-70 \mathrm{mV})$ of the action potential.

Fig.4 shows the simulation results and clearly the numerical 


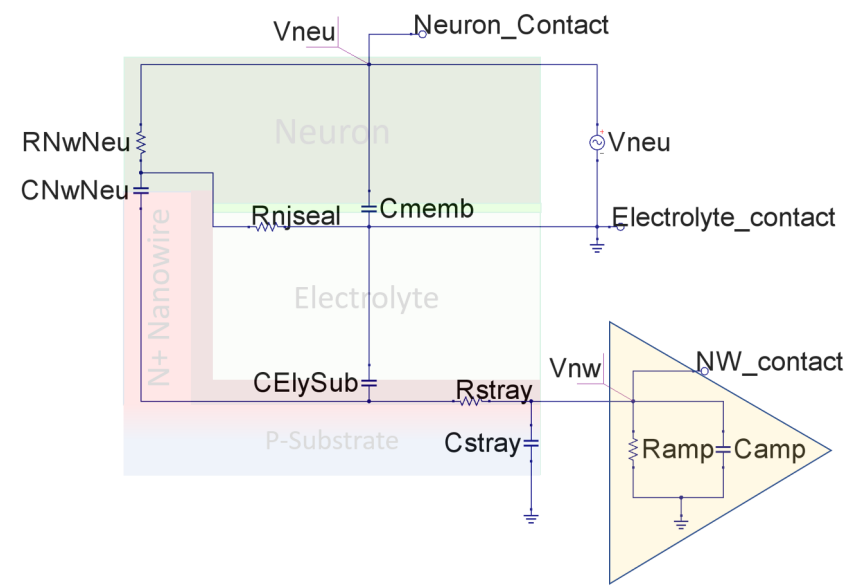

Fig. 3. Simplified lumped-element equivalent circuit model during intracellular access. $C_{m e m b}$ is the neuron membrane capacitance; $R_{N w N e u}$ is the electrolyte resistance between the neuron and the tip of the NW; $R_{n j s e a l}$ is the nano-junctional sealing resistance; $C_{N w N e u}$ is the EDL capacitance of the intracellular electrolyte toward the NW; $C_{E l y S u b}$ is the insulator capacitance between the electrolyte and the substrate; $R_{\text {stray }}$ and $C_{\text {stray }}$ account for the parasitic resistance and capacitance of the interconnects; $R_{a m p}$ and $C_{a m p}$ are accounting for the input impedance of the readout.

\begin{tabular}{|c|c|}
\hline$C_{\text {memb }}$ & $C_{\text {memb }}\left[\frac{F}{\mathrm{~cm}^{2}}\right]$ Area \\
\hline$R_{N w N e u}$ & $\rho_{\text {neu }} \frac{h_{n e u}}{\text { section }_{N W}}$ \\
\hline$C_{N w N e u}$ & $C_{E D L, n e u}\left[\frac{F}{\mathrm{~cm}^{2}}\right]$ Area \\
\hline$C_{\text {ElySub }}$ & $C_{\text {insulator }}\left[\frac{F}{\text { cm }^{2}}\right]$ Area \\
\hline$R_{\text {stray }}$ & $\rho_{\text {lines }} \frac{\text { length }_{\text {lines }}}{\text { section }}$ lines $_{\text {lines }}$ \\
\hline$C_{\text {stray }}$ & $\frac{\epsilon_{\text {insulator }} \text { surface }_{\text {lines }}}{\text { thickness }_{\text {insulator }}}$ \\
\hline
\end{tabular}

TABLE II

RELATIONS BETWEEN THE LUMPED COMPONENTS AND THE PHYSICAL-GEOMETRICAL PARAMETERS OF THE STRUCTURE

and lumped element models appear in excellent agreement, thus confirming that the reduced complexity of the model in Fig. 3 does not affect its ability to reproduce the essential physics of the system. The lumped model, with component values analytically expressed in terms of geometrical and physical properties ot the materials, allows users to quickly understand how the physics and the geometry variables of the structure affect the lumped components and the response.

\section{TRANFER FUNCTION AND SNR}

Given the accuracy achieved by the simpler model, we used it to study the neuron to readout transfer function $G(j \omega)=$

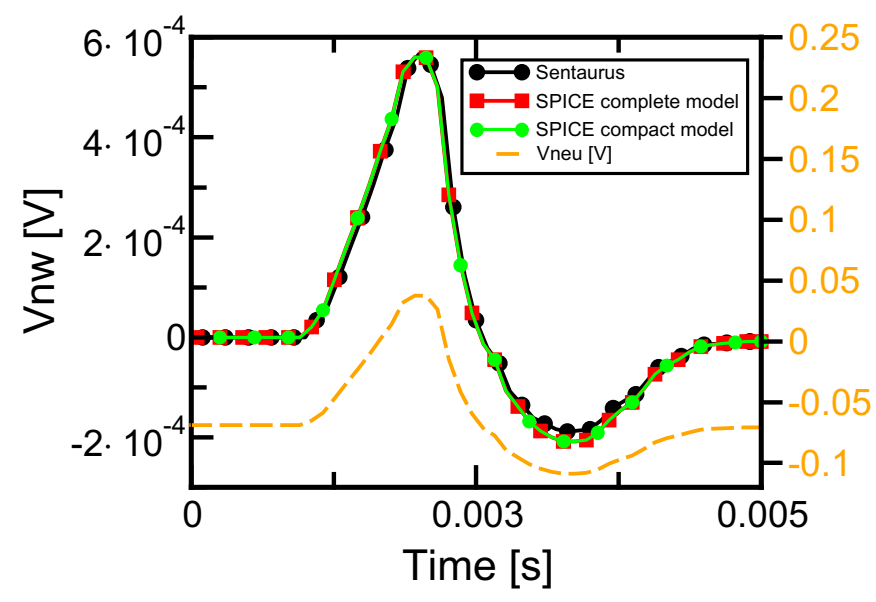

Fig. 4. Comparison between Sentaurus (FEM) transient simulation and SPICE (lumped circuit model) transient simulation with complete and compact models. In this case we set $l v l_{n e u}=1 \mu m$ meaning that the neuron is only in contact with the NW-tip, as depicted in Fig. 2.

$V_{n w}(j \omega) / V_{\text {neu }}(j \omega)$ of the system. The transfer function has been calculated firstly in symbolic form:

$$
G(j \omega)=\frac{\frac{R_{n j s e a l}}{R_{N w N e u}+R_{n j s e a l}} j \omega C_{N w N e u} R_{a m p}}{1+j \omega \alpha-\omega^{2} \beta-j \omega^{3} \gamma}
$$

where:

$$
\begin{aligned}
& \alpha=C_{E L y S u b}\left(R_{a m p}+R_{\text {stray }}\right)+C_{N w N e u}\left(R_{a m p}+R_{\text {stray }}+\right. \\
& \left.R_{\text {njseal }} / / R_{N w N e u}\right)+\left(C_{a m p}+C_{\text {stray }}\right) R_{a m p} \\
& \beta=C_{E l y S u b} C_{N w N e u}\left(R_{a m p}+R_{\text {stray }}\right)\left[R_{n j \text { seal }} / / R_{N w N e u}\right]+ \\
& C_{\text {ElySub }}\left(C_{a m p}+C_{\text {stray }}\right) R_{a m p} R_{\text {stray }}+\left(C_{a m p}+C_{\text {stray }}\right) \\
& \text {. } C_{N w N e u} R_{a m p}\left(R_{\text {stray }}+R_{\text {njseal }} / / R_{N w N e u}\right) \\
& \gamma=C_{\text {ElySub }} C_{N w N e u}\left(C_{a m p}+C_{\text {stray }}\right) R_{a m p} R_{\text {stray }}\left[R_{n j s e a l} / / R_{N w N e u}\right]
\end{aligned}
$$

It comprises three-poles and a zero in the origin. Unfortunately the unfactorized polynomial form of Eq. (1) is practically useless, since it does not separate the contribution each component is giving to the frequency response.

To cast $G(j \omega)$ in an insightful form, we carried out opencircuit and short-circuit time constant analysis to find out the highest- and lowest- frequency poles, respectively. Then, the pole that falls in between these two is extracted analytically from Eq. (1). The transfer function in factorized form is:

$$
G(j \omega)=\frac{\frac{R_{n j s e a l}}{R_{N w N e u}+R_{n j s e a l}} j \omega C_{N w N e u} R_{a m p}}{(1+j \omega a)(1+j \omega b)(1+j \omega c)}
$$

where:

$$
\begin{aligned}
& a=\frac{1}{2 \pi f_{p 1}}=\left(C_{N w N e u}+C_{a m p}+C_{\text {stray }}+C_{\text {ElySub }}\right) R_{a m p} \\
& b=\frac{1}{2 \pi f_{p 2}}=C_{\text {ElySub }}\left(R_{N w N e u} / / R_{\text {stray }}\right) \\
& c=\frac{1}{2 \pi f_{p 3}}=C_{N w N e u}\left(R_{\text {stray }}+R_{N w N e u} / / R_{\text {njseal }}\right)
\end{aligned}
$$


It is then straightforward to identify the in-band gain, poles and zero expressions, as shown in the graphical representation of Fig. 5 (blue curve). In particular,

$$
\text { Gain }_{\text {in-band }}=\frac{\frac{R_{n j s e a l}}{R_{N w N e u}+R_{n j s e a l}} C_{N w N e u}}{C_{N w N e u}+C_{a m p}+C_{\text {stray }}+C_{E l y S u b}}
$$

Numerical examples of the poles and the in-band gain values

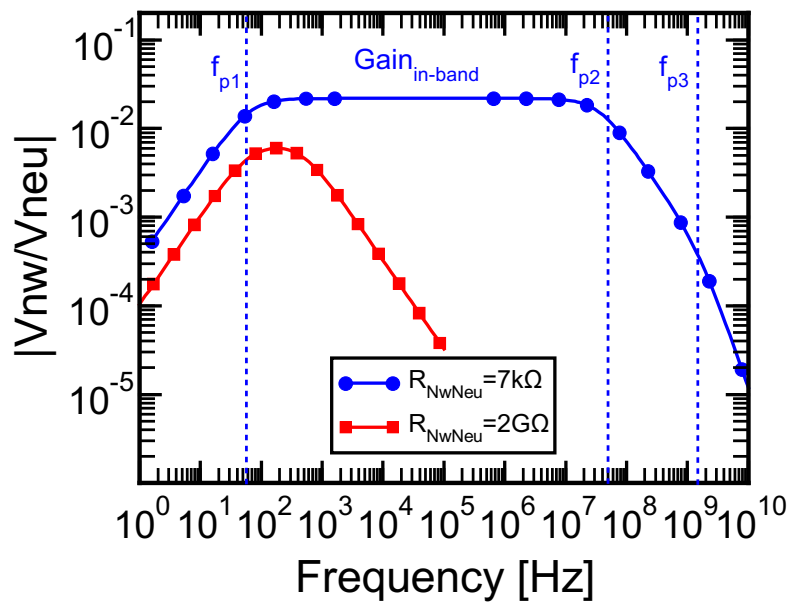

Fig. 5. Factorized transfer function with explicit identification of the poles and the in-band gain on the ideal membrane penetration case curve (blue curve). The red curve represents the case of real membrane penetration that leads to an experimental $R_{N w N e u}$ of $2 \Omega$ [16]. In both cases the $l v l_{n e u}=1 \mu \mathrm{m}$ meaning the neuron is only in contact with the NW-tip, as depicted in Fig. 2.

encountered in simulation are shown in Table III, for needles fully and partially penetrating the neuron.

It is interesting to note that the in-band gain, Eq. (3), is set by the resistive divider between $R_{n j s e a l}$ and $R_{N w N e u}$ (which is maximized if the neuron adheres to the nanowire thus providing good sealing of the contact tip and good nanowire penetration [16]) and the capacitive divider between the neuron-nanowire coupling capacitance $C_{N w N e u}$ and all the other capacitances affecting the output node (NW_contact).

To maximize this term the needle should penetrate well into the neuron, the needle doping should be in the $10^{19} \mathrm{~cm}^{-3}$ order of magnitude or even better be siliciurized in order to avoid depletion. The stray capacitance as well as the input capacitance of the readout should be small.

As regards the signal to noise ratio, SNR, here defined as the peak-to-peak AP amplitude divided by the rms output voltage: $V_{n w}[V p p] / V_{N}[V]$, some assumptions have been made to compute the noise output voltage. In particular we consider only the thermal noise due to the semiconductor, the electrolyte, the parasitics of the interconnect and the readout resistances, neglecting any other source of biological or 1/f noise, which of course might be present [17]. Therefore the calculations at present yields only a best case estimate of the SNR. To evaluate the SNR at the input of the readout, we firstly consider the output noise directly extracted from SPICE AC simulations at the NW_contact node employing a $1 \mathrm{MHz}$ bandwidth, a reasonable value for the readout circuits of neuronal signals

\begin{tabular}{|c|c|c|}
\hline $\begin{array}{l}\left(l v l_{\text {neu }}=40 \mathrm{~nm}\right) \\
\text { Needle fully } \\
\text { inserted in the } \\
\text { neuron }\end{array}$ & $\begin{array}{llr}R_{\text {amp }}= & 100 G \Omega \\
C_{\text {amp }}= & 10 p F \\
R_{\text {stray }}= & 1.8 \mathrm{k} \Omega \\
C_{\text {stray }}= & 13.8 p F \\
R_{\text {njseal }}= & 900 \mathrm{M} \Omega \\
R_{\text {NwNeu }}= & 700 \Omega \\
C_{\text {NwNeu }}= & 2.9 p F \\
C_{\text {ElySub }}=54 \mathrm{fF}\end{array}$ & $\begin{array}{llr}f_{p 1}= & 0.059 \mathrm{~Hz} \\
f_{p 2}= & 26 \mathrm{MHz} \\
f_{p 3}= & 7.3 \mathrm{GHz} \\
\text { Gain }_{\text {in-band }}= & 0.108\end{array}$ \\
\hline $\begin{array}{l}\left(l v l_{\text {neu }}=1 \mu m\right) \\
\text { Neuron in } \\
\text { contact with the } \\
\text { needle tip }\end{array}$ & $\begin{array}{llr}R_{\text {amp }}= & 100 \mathrm{G} \Omega \\
C_{\text {amp }}= & 10 \mathrm{pF} \\
R_{\text {stray }}= & 1.8 \mathrm{k} \Omega \\
C_{\text {stray }}= & 13.8 \mathrm{pF} \\
R_{\text {njseal }}= & 900 \mathrm{M} \Omega \\
R_{\text {NwNeu }}= & 7 \mathrm{k} \Omega \\
C_{\text {NwNeu }}= & 610 \mathrm{fF} \\
C_{\text {ElySub }}= & 59 \mathrm{fF}\end{array}$ & $\begin{array}{llr}f_{p 1}= & 0.065 \mathrm{~Hz} \\
f_{p 2}= & 38 \mathrm{MHz} \\
f_{p 3}= & 2.0 \mathrm{GHz} \\
\text { Gain }_{\text {in-band }}= & 0.025\end{array}$ \\
\hline $\begin{array}{l}\left(l v l_{\text {neu }}=1 \mu m\right) \\
\text { Neuron } \\
\text { partially in } \\
\text { contact with } \\
\text { the needle tip } \\
\text { (membrane } \\
\text { poration [16]) }\end{array}$ & $\begin{array}{llr}R_{\text {amp }}= & 100 \mathrm{G} \Omega \\
C_{\text {amp }}= & 10 \mathrm{pF} \\
R_{\text {stray }}= & 1.8 \mathrm{k} \Omega \\
C_{\text {stray }}= & 13.8 \mathrm{pF} \\
R_{\text {njseal }}= & 900 \mathrm{M} \Omega \\
R_{\text {NwNeu }}= & 2 \mathrm{G} \Omega \\
C_{\text {NwNeu }}= & 610 \mathrm{fF} \\
C_{\text {ElySub }}= & 59 \mathrm{fF}\end{array}$ & $\begin{array}{lrr}f_{p 1}= & 0.065 \mathrm{~Hz} \\
f_{p 2}= & 429 \mathrm{~Hz} \\
f_{p 3}= & 1.5 \mathrm{GHz} \\
\text { Gain }_{\text {in-band }}= & 0.008\end{array}$ \\
\hline
\end{tabular}

TABLE III

POLES AND IN-BAND GAIN NUMERICAL VALUES

given the time scale of such signals.

The result is plotted in Fig.6 for two cases: one where $R_{a m p}=100 \mathrm{G} \Omega$ (as reported for instance in [16]) as main thermal noise source of the overall circuit (black line of Fig. 6 ), and one where the $R_{a m p}$ thermal noise contribution is artificially suppressed to highlight the noise generated by the electrolyte, the needle and the interconnections only (red line of Fig. 6).

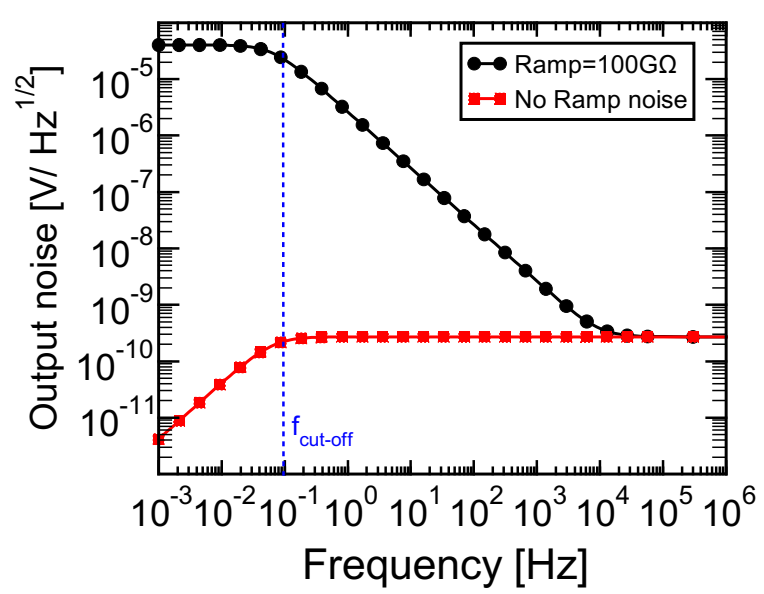

Fig. 6. Output noise of the circuit with and without the thermal noise of $R_{\text {amp }}=100 \mathrm{G} \Omega$. In this case we set $l v l_{n e u}=1 \mu \mathrm{m}$ meaning that the neuron is only in contact with the NW-tip, as depicted in Fig. 2.

As depicted in Fig. 6, the cut-off frequency of the thermal noise is the same with and without the $R_{a m p}$ noise contribution 
and is given by :

$$
f_{\text {cut-off }}=\frac{1}{2 \pi R_{a m p}\left(C_{\text {stray }}+C_{a m p}\right)}
$$

The final consideration concerns how much the $C_{\text {load }}=C_{\text {amp }}+C_{\text {stray }}$ reduces the noise and consequently how much it will affect the SNR, since, whenever the dominant thermal noise of the overall system is due to $R_{a m p}$, the following noise expression holds [18]:

$$
V_{N}=\sqrt{\frac{K T}{C_{\text {load }}}} .
$$

Fig. 6 depicts the trend of the SNR for different values of $C_{\text {load }}$. It clearly shows that the lower the $C_{\text {load }}$ the better, since

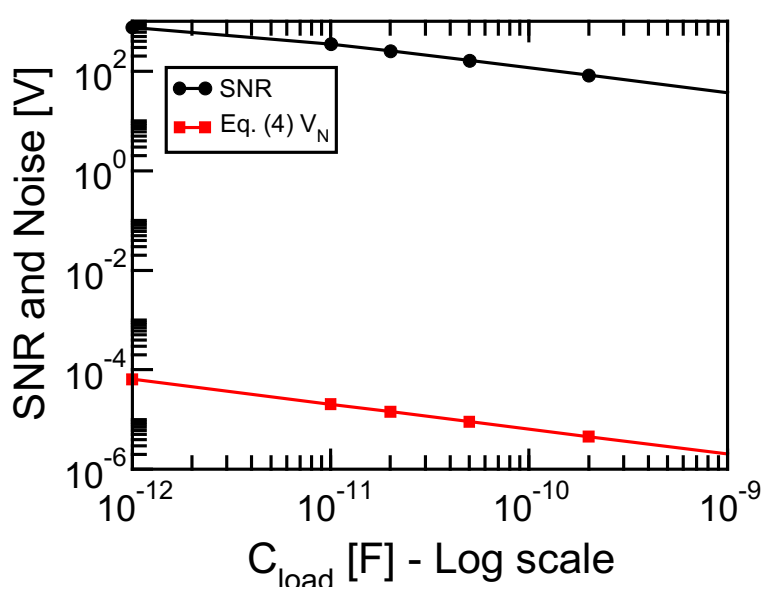

Fig. 7. SNR of the device as function of $C_{\text {load }}$. In this case we $l v l_{n e u}=1$ $\mu m$ meaning that the neuron is only in contact with the NW-tip, as depicted in Fig. 2.

the improvement in terms of gain is larger compared to the corresponding worsening of the noise level.

\section{CONCLUSION}

The joint use of numerical and lumped-element equivalent circuit models outlines a methodology to reliably and efficiently describe at circuit level the physics of the neuronnanoneedle system under study. The lumped-element model takes less simulation time while still retaining a direct link with physical and geometrical design parameters. The lumped components affect the overall transfer function and the model highlights their role and supports the design based on the specifications of the readout circuit.

The models are helpful to interpret experimental results as well, in particular to extract (by fitting) the experimental variables that depend on the random nature of the neuron adherence to the nanowire (such as $R_{\text {njseal }}$ ).

The SNR evaluation, although still limited by the lack of noise generation mechanisms besides thermal noise, is useful for device-optimization and for comparison among different transduction mechanisms (voltage, current). In perspective the lumped-element circuit could be enriched by introducing the membrane conductances as originally proposed in [15].

\section{ACKNOWLEDGMENT}

This work was supported in part by the European Commission H2020 project IN-FET (g.a. 862882) via the IUNET consortium.

\section{REFERENCES}

[1] R. Liu et al., "High Density Individually Addressable Nanowire Arrays Record Intracellular Activity from Primary Rodent and Human Stem Cell Derived Neurons", Nano lett. 17 (5), 2757-2764, 2017.

[2] J. Abbot at al., "A nanoelectrode array for obtaining intracellular recordings from thousands of connected neurons", Nat Biomed Eng 4, 232-241, 2019.

[3] J. Abbott et al., "CMOS nanoelectrode array for all-electrical intracellular electrophysiological imaging", Nature Nanotech 12, 460-466, 2017.

[4] A. Casanova et al., "Integration of FinFETs and 3D nanoprobes devices on a common bio-platform for monitoring electrical activity of single neurons", IEEE International Electron Devices Meeting (IEDM), San Francisco, CA, pp. 26.4.1-26.4.4, 2017.

[5] M. Dipalo et al., "Intracellular and Extracellular Recording of Spontaneous Action Potentials in Mammalian Neurons and Cardiac Cells with 3D Plasmonic Nanoelectrodes", Nano lett. 17 (6), 3932-3939, 2017.

[6] M.E. Spira, A. Hai, "Multi-electrode array technologies for neuroscience and cardiology", Nature Nanotech. 8 (2), 83-94, 2013.

[7] A. M Ojovan et al., "A feasibility study of multi-site,intracellular recordings from mammalian neurons by extracellular gold mushroom-shaped microelectrodes", Scientific Reports 5 (14100), 2015.

[8] P.D. Jones et al., "Low-Impedance 3D PEDOT:PSS Ultramicroelectrodes", Frontiers in Neuroscience 14 (405), 2020.

[9] https://www.synopsys.com/silicon/tcad/device-simulation/sentaurusdevice.html

[10] A. Bandiziol et al., "A TCAD-Based Methodology to Model the SiteBinding Charge at ISFET/Electrolyte Interfaces", IEEE Trans. Electron Device, 62, 3379-3386, 2015.

[11] J. J. Harris et al., "Energy-Efficient Information Transfer by Visual Pathway Synapses", Current Biology, 25, 3151-3160, 2015.

[12] V. Gautam et al., "Engineering highly interconnected neuronal networks on nanowire scaffolds", NANO lett. 17 (6), 3369 - 3375, 2017.

[13] J.R. Buitenweg et al., "Finite element modeling of the neuron-electrode interface" IEEE engineering in medicine and biology magazine, 46-52, 2000.

[14] P. Massobrio et al., "Interfacing Cultured Neurons to Microtransducers Arrays: A Review of the Neuro-Electronic Junction Models",Frontiers in Neuroscience, 10, 282, 2016.

[15] M. Storace et al., "Simulations of the Behavior of Synaptically Driven Neurons Via Time-Invariant Circuit Models",IEEE Trans. Biomed. Eng., 4 (12), 1282-1287, 1997.

[16] L. Guo, "On neural recording using nanoprotrusion electrodes", J. Neural Eng., 17, 016017, 2020.

[17] L.J. Mele et al., "A model of the interface charge and chemical noise due to surface reactions in Ion Sensitive FETs", SISPAD, 1-4, 2019.

[18] B.Razavi, RF Microelectronics, 2nd edition, Prentice Hall, 2012. 


\section{APPENDIX}

Fig. A1 reports the complete equivalent lumped-element circuit model of the structure. The electrolyte and multiple $\mathrm{N}^{+}$region at the basis of the nanowire are represented by multiple RC cells to account for their distributed nature resulting from the large dimension compared to those of the nanowire.

Adopting the terminology in [16]: $\mathrm{nm}=$ non-junctionalmembrane (neuron membrane that does not face the electrode), jm=junctional-membrane (neuron membrane that faces the electrode), njm=nano-junctional-membrane (neuron membrane in correspondence of the NW), the physical meaning of the lumped elements is as follows:

$-\mathrm{V}_{\text {neu_nm }}$ is the AP voltage generator applied on the nm-side of the neuron.

$-\mathrm{R}_{\mathrm{nm}}$ is the $\mathrm{nm}$-conductance.

$-\mathrm{C}_{\mathrm{nm}}$ is the $\mathrm{nm}$-capacitance.

$-\mathrm{R}_{\mathrm{Neu} \_n m}$ is the fraction of intracellular electrolyte resistance from the nm-side to the centre of the cell.

$-\mathrm{C}_{\mathrm{Neu} \_n \mathrm{~m}}$ is the fraction of intracellular electrolyte capacitance from the nm-side to the centre of the cell.

$-\mathrm{R}_{\mathrm{Neu} \_ \text {nim }}$ is the fraction of intracellular electrolyte resistance from the njm-side to the centre of the cell.

$-\mathrm{C}_{\mathrm{Neu} \_n j m}$ is the fraction of intracellular electrolyte capacitance from the njm-side to the centre of the cell.

$-\mathrm{R}_{\mathrm{Neu} \_\mathrm{jm}}$ is the fraction of intracellular electrolyte resistance from the jm-side to the centre of the cell.

$-\mathrm{C}_{\mathrm{Neu} \_\mathrm{jm}}$ is the fraction of intracellular electrolyte capacitance from the jm-side to the centre of the cell.

$-\mathrm{V}_{\text {neu_jm }}$ is the AP voltage generator applied on the jm-side of the neuron.

$-R_{j m}$ is the jm-conductance.

$-\mathrm{C}_{\mathrm{jm}}$ is the jm-capacitance.

$-\mathrm{R}_{\mathrm{njseal}}$ is the nj-sealing resistance between the NW and the neuron.

$-\mathrm{C}_{\mathrm{NwNeu}}$ is the intracellular electrolyte EDL coupling capacitance toward the NW-tip.

$-\mathrm{R}_{\mathrm{NW} \text { cyl_half1 }}, \mathrm{R}_{\mathrm{NW} \text { cyl_half2 }}$ are the two half of the nanowire resistance (only the pillar resistance).

-R R $\mathrm{R}_{\text {Nbase_half1 }}, \quad \mathrm{R}_{\text {NWbase_half2_third1 }}, \quad \mathrm{R}_{\text {NWbase_half2_third2, }}$ $\mathrm{R}_{\mathrm{NWbase} \text { half2_third3 }}$ are the distributed resistances of the base of the nanowire (of the electrode).

$-\mathrm{C}_{\text {dep_NW }}$ is the junction capacitance underlying the NW cross-section.

$-\mathrm{C}_{\text {dep_base_third1 }}, \mathrm{C}_{\text {dep_base_third2 }}, \mathrm{C}_{\text {dep_base_third3 }}$ are distributed the junction capacitances underlying the whole nanowire base excluding the $\mathrm{C}_{\text {dep_Nw }}$ contribution.

$-\mathrm{C}_{\mathrm{NWEly}}$ is the coupling capacitance between the extracellular electrolyte and the NW.

-Rely_third1, REly_third2, REly_third3_third1, Rely_third3_third2, $R_{\text {Ely_third3_third3 }}$ are the distributed extracellular electrolyte resistances.

$-R_{\text {Ely_ext }}$ is the external lumped electrolyte resistance introduced to keep the simulation domain at manageable size. $-R_{\text {Ely_nm }}$ is the fraction of extracellular electrolyte resistance from the nm-side to the far bulk electrolyte contact.

$-\mathrm{C}_{\text {Ely_nm }}$ is the fraction of extracellular electrolyte capacitance from the nm-side to the far bulk electrolyte contact.

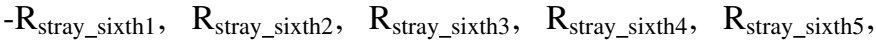
$\mathrm{R}_{\text {stray_sixth6 }}$ are the distributed parasitic resistances of the interconnects.

$-\mathrm{C}_{\text {stray_sixth1 } 1}, \mathrm{C}_{\text {stray_sixth2 }}, \mathrm{C}_{\text {stray_sixth } 3}, \mathrm{C}_{\text {stray_sixth4 }}, \mathrm{C}_{\text {stray_sixth5 }}$, $\mathrm{C}_{\text {stray_sixth6 }}$ are the distributed parasitic capacitances of the interconnects.

$-R_{a m p}$ is the input resistance of the readout.

$-\mathrm{C}_{\mathrm{amp}}$ is the input capacitance of the readout.

Notice: $\mathrm{C}_{\text {Ely }}$ is neglected since at low frequency its contribution is negligible.

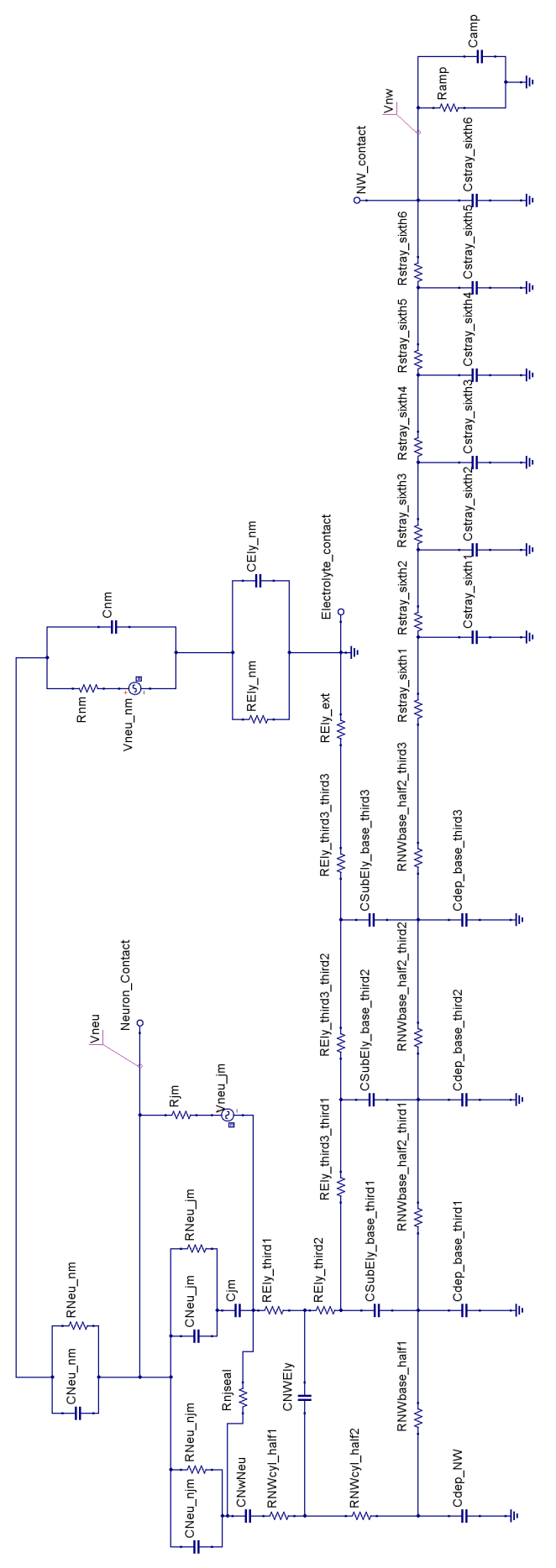


Fig. A2 shows the nanowire-neuron coupling capacitance for different NW doping and shapes, as a function of the applied $\mathrm{DC}$ value of $\mathrm{V}_{\text {neu. }}$.

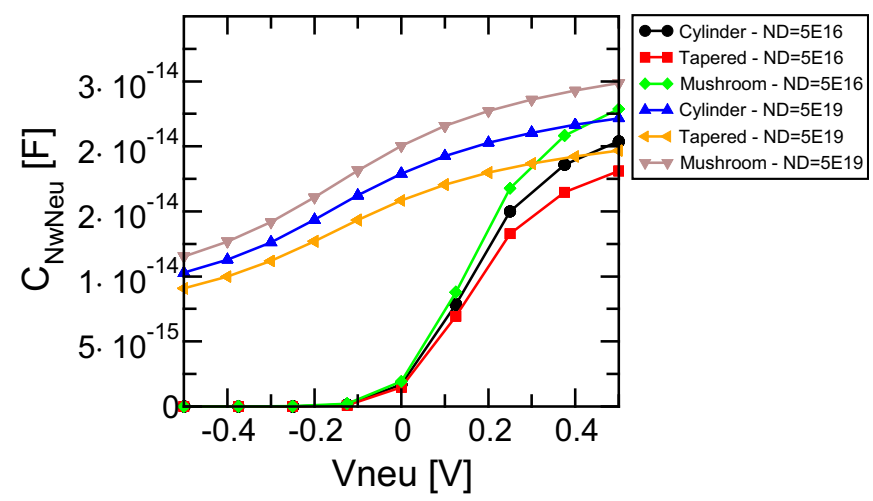

